PROCEEDINGS OF THE

AMERICAN MATHEMATICAL SOCIETY

Volume 128, Number 8, Pages 2373-2379

S 0002-9939(00)05644-6

Article electronically published on February 23, 2000

\title{
MULTIPLE SYMMETRIC POSITIVE SOLUTIONS FOR A SECOND ORDER BOUNDARY VALUE PROBLEM
}

\author{
JOHNNY HENDERSON AND H. B. THOMPSON
}

(Communicated by Hal L. Smith)

\begin{abstract}
For the second order boundary value problem, $y^{\prime \prime}+f(y)=0$, $0 \leq t \leq 1, y(0)=0=y(1)$, where $f: \mathbb{R} \rightarrow[0, \infty)$, growth conditions are imposed on $f$ which yield the existence of at least three symmetric positive solutions.
\end{abstract}

\section{Introduction}

In this paper, we are concerned with the existence of multiple solutions for the second order boundary value problem

$$
\begin{aligned}
& y^{\prime \prime}+f(y)=0, \quad 0 \leq t \leq 1, \\
& y(0)=0=y(1),
\end{aligned}
$$

where $f: \mathbb{R} \rightarrow[0, \infty)$ is continuous. A solution $y \in C^{(2)}[0,1]$ of (1.1), (1.2) is both nonnegative and concave on $[0,1]$. We will impose growth conditions of $f$ which ensure the existence of at least three symmetric positive solutions of (1.1), (1.2).

Our results can be thought of as an extension of those by Avery [2], Leggett and Williams [6], and Guo and Lakshmikantham [4], in which they obtained three positive solutions of (1.1), (1.2). Our ability to obtain symmetric solutions arises from the symmetry of an associated Green's function.

Other attention has been given to triple solutions in the paper by Anderson [1] for three-point boundary value problems for third order equations, as well as in the paper by Henderson and Thompson [5] for certain two-point boundary value problems for $n$-th order equations. Each of the papers [1, 22] and [5] makes an application of a fixed point theorem by Leggett-Williams [6], which they developed using the fixed point index in order Banach spaces. Leggett-Williams [6] applied their fixed point theorem to prove the existence of three positive solutions for Hammerstein integral equations of the form, $y=\int_{\Omega} G(x, s) f(s, y(s)) d s, \Omega \subset \mathbb{R}^{n}$, by making use of suitable inequalities they imposed on the kernel $G$ and on $f$. Sun and Sun [7] gave an extension of the Leggett-Williams fixed point theorem for multiple fixed points, and then Avery and Peterson [3] applied the Sun-Sun extension to obtain three positive solutions of boundary value problems for second order difference equations.

Received by the editors September 19, 1998.

2000 Mathematics Subject Classification. Primary 34B15.

Key words and phrases. Boundary value problem, Green's function, multiple solutions.

(C)2000 American Mathematical Society 
In Section 2, we provide some background results, and we state the LeggettWilliams fixed point theorem. Then, in Section 3, we impose growth conditions on $f$ which allow us to apply the Leggett-Williams fixed point theorem in obtaining three symmetric positive solutions of (1.1), (1.2).

\section{Some BACKGRound DEFInitions AND RESUlts}

In this section, we provide some background material from the theory of cones in Banach spaces, in order that this paper be self-contained. We also state a fixed point theorem due to Leggett and Williams [6] for multiple fixed points of a cone preserving operator.

If $\mathfrak{P} \subset \mathfrak{B}$ is a cone, we denote the order induced by $\mathfrak{P}$ on $\mathfrak{B}$ by $\leq_{\mathfrak{P}}$.

Definition. A map $\alpha$ is said to be a nonnegative continuous concave functional on $\mathfrak{P}$ if $\alpha: \mathfrak{P} \rightarrow[0, \infty)$ is continuous and

$$
\alpha(t x+(1-t) y) \geq t \alpha(x)+(1-t) \alpha(y),
$$

for all $x, y \in \mathfrak{P}$ and $0 \leq t \leq 1$.

Definition. For numbers $0<a<b$ and $\alpha$ a nonnegative continuous concave functional on $\mathfrak{P}$, define convex sets $\mathfrak{P}_{r}$ and $\mathfrak{P}(\alpha, a, b)$ respectively by

$$
\mathfrak{P}_{r}=\{y \in \mathfrak{P} \mid\|y\|<r\}
$$

and

$$
\mathfrak{P}(\alpha, a, b)=\{y \in \mathfrak{P} \mid a \leq \alpha(y),\|y\| \leq b\} .
$$

In obtaining multiple symmetric positive solutions of (1.1), (1.2), the following fixed point theorem of Leggett and Williams will be fundamental.

Theorem 2.1. Let $A: \overline{\mathfrak{P}}_{c} \rightarrow \overline{\mathfrak{P}}_{c}$ be completely continuous and $\alpha$ be a nonnegative continuous concave functional on $\mathfrak{P}$ such that $\alpha(y) \leq\|y\|$, for all $y \in \overline{\mathfrak{P}}_{c}$. Suppose there exist $0<a<b<d \leq c$ such that

(C1) $\{y \in \mathfrak{P}(\alpha, b, d) \mid \alpha(y)>b\} \neq \emptyset$ and $\alpha(A y)>b$, for $y \in \mathfrak{P}(\alpha, b, d)$,

(C2) $\|A y\|<a$, for $\|y\| \leq a$, and

(C3) $\alpha(A y)>b$, for $y \in \mathfrak{P}(\alpha, b, c)$ with $\|A y\|>d$.

Then $A$ has at least three fixed points, $y_{1}, y_{2}$ and $y_{3}$ satisfying

$$
\left\|y_{1}\right\|<a, b<\alpha\left(y_{2}\right) \text {, and }\left\|y_{3}\right\|>a \text { with } \alpha\left(y_{3}\right)<b .
$$

\section{Multiple symmetric Positive solutions}

In this section, we will impose growth conditions on $f$ which allow us to apply Theorem 2.1 in regard to obtaining three symmetric positive solutions of (1.1) (1.2). We will apply Theorem 2.1 in conjunction with a completely continuous operator whose kernel $G(t, s)$ is the Green's function for

$$
-y^{\prime \prime}=0
$$

satisfying (1.2). In particular,

$$
G(t, s)= \begin{cases}t(1-s), & 0 \leq t \leq s \leq 1 \\ s(1-t), & 0 \leq s \leq t \leq 1\end{cases}
$$


We will make use of various properties of $G(t, s)$ which include

$$
\begin{aligned}
& 0<G(t, s) \leq G(s, s)=s(1-s), 0<t, s<1, \\
& G(t, s) \geq \frac{1}{4} G(s, s)=\frac{1}{4} s(1-s), \frac{1}{4} \leq t \leq \frac{3}{4}, 0 \leq s \leq 1, \\
& \max _{0 \leq t \leq 1} \int_{0}^{1} G(t, s) d s=\frac{1}{8}, \\
& \min _{\frac{1}{4} \leq t \leq \frac{3}{4}} \int_{\frac{1}{4}}^{\frac{3}{4}} G(t, s) d s=\int_{\frac{1}{4}}^{\frac{3}{4}} G\left(\frac{1}{4}, s\right) d s=\frac{1}{16}, \text { and } \\
& \min _{0 \leq r \leq 1} \frac{G\left(\frac{1}{4}, r\right)}{G\left(\frac{1}{2}, r\right)}=\frac{1}{2} .
\end{aligned}
$$

Next, let $\mathfrak{B}=C[0,1]$ be endowed with the maximum norm, $\|y\|=\max _{0 \leq t \leq 1}|y(t)|$, and the ordering $x \leq y$ if $x(t) \leq y(t)$ for all $t \in[0,1]$. Let the cone $\mathfrak{P} \subset \mathfrak{B}$ of symmetric, positive functions in $\mathfrak{B}$ define this ordering so that

$$
\mathfrak{P}=\{y \in \mathfrak{B} \mid y \text { is concave, symmetric and nonnegative valued on }[0,1]\} \text {. }
$$

Finally, let the nonnegative continuous concave functional $\alpha: \mathfrak{P} \rightarrow[0, \infty)$ be defined by

$$
\alpha(y)=\min _{\frac{1}{4} \leq t \leq \frac{3}{4}} y(t), \quad y \in \mathfrak{P} .
$$

We observe here that, for each $y \in \mathfrak{P}$,

$$
\alpha(y)=y\left(\frac{1}{4}\right) \leq y\left(\frac{1}{2}\right)=\|y\|
$$

and also that $y \in \mathfrak{B}$ is a solution of (1.1), (1.2) if and only if

$$
y(t)=\int_{0}^{1} G(t, s) f(y(s)) d s, \quad 0 \leq t \leq 1 .
$$

We now present our result of the paper.

Theorem 3.1. Let $0<a<b<c / 2$, and suppose $f$ satisfies

(i) $f(w)<8 a$, for $0 \leq w \leq a$,

(ii) $f(w) \geq 16 b$, for $b \leq w \leq 2 b$, and

(iii) $f(w) \leq 8 c$, for $0 \leq w \leq c$.

Then, the boundary value problem (1.1), (1.2) has three symmetric positive solutions $y_{1}, y_{2}$ and $y_{3}$ satisfying $\left\|y_{1}\right\|<a, b<\alpha\left(y_{2}\right)$, and $\left\|y_{3}\right\|>a$ with $\alpha\left(y_{3}\right)<b$.

Proof. We begin by defining a completely continuous operator $A: \mathfrak{B} \rightarrow \mathfrak{B}$ by

$$
A y(t)=\int_{0}^{1} G(t, s) f(y(s)) d s .
$$

We seek fixed points of $A$ which satisfy the conclusion of the theorem. We note first, if $y \in \mathfrak{P}$, then from properties of $G(t, s), A y(t) \geq 0$ and $(A y)^{\prime \prime}(t)=-f(y(t)) \leq 0$, $0 \leq t \leq 1$, and $A y(t)=A y(1-t), 0 \leq t \leq \frac{1}{2}$, and consequently, $A y \in \mathfrak{P}$; that is, $A: \mathfrak{P} \rightarrow \mathfrak{P}$. 
We now show that the conditions of Theorem 2.1 are satisfied. We noted in (3.9) that $\alpha(y) \leq\|y\|$, for all $y \in \mathfrak{P}$. Now choose $y \in \mathfrak{P}_{c}$. Then $\|y\| \leq c$ and assumption (iii) yields $f(y(s)) \leq 8 c, 0 \leq s \leq 1$. Thus, from (3.5)

$$
\begin{aligned}
\|A y\| & =\max _{0 \leq t \leq 1} \int_{0}^{1} G(t, s) f(y(s)) d s \\
& \leq \max _{0 \leq t \leq 1} \int_{0}^{1} G(t, s) 8 c d s \\
& =c .
\end{aligned}
$$

Hence, $A: \overline{\mathfrak{P}}_{c} \rightarrow \overline{\mathfrak{P}}_{c}$. In a similar vein, fulfillment of condition (C2) of Theorem 2.1 is completed. That is, if $y \in \overline{\mathfrak{P}}_{a}$, then assumption (i) yields $f(y(s))<8 a$, $0 \leq s \leq 1$, from which we obtain, as in the argument above, that $A: \overline{\mathfrak{P}}_{a} \rightarrow \mathfrak{P}_{a}$.

To fulfill property $(\mathrm{C} 1)$ of Theorem $[2.1$ we note that $x(t)=2 b, 0 \leq t \leq 1$, is a member of $\mathfrak{P}(\alpha, b, 2 b)$ and $\alpha(x)=\alpha(2 b)>b$, and so $\{y \in \mathfrak{P}(\alpha, b, 2 b) \mid \alpha(y)>$ $b\} \neq \emptyset$. In addition, if we choose $y \in \mathfrak{P}(\alpha, b, 2 b)$, then $\alpha(y)=y\left(\frac{1}{4}\right) \geq b$, and so $b \leq y(s) \leq 2 b, \frac{1}{4} \leq s \leq \frac{3}{4}$. Thus, for any $y \in \mathfrak{P}(\alpha, b, 2 b)$, assumption (ii) yields $f(y(s)) \geq 16 b \frac{1}{4} \leq s \leq \frac{3}{4}$, and consequently, from (3.6)

$$
\begin{aligned}
\alpha(A y) & =\min _{\frac{1}{4} \leq t \leq \frac{3}{4}} A y(t) \\
& =\int_{0}^{1} G\left(\frac{1}{4}, s\right) f(y(s)) d s \\
& >\int_{\frac{1}{4}}^{\frac{3}{4}} G\left(\frac{1}{4}, s\right) f(y(s)) d s \\
& \geq \int_{\frac{1}{4}}^{\frac{3}{4}} G\left(\frac{1}{4}, s\right) 16 b d s \\
& =b .
\end{aligned}
$$

Hence, condition (C1) of Theorem 2.1 is satisfied. We finally exhibit that (C3) of Theorem [2.1] is also satisfied. (In particular, we show, if $y \in \mathfrak{P}(\alpha, b, c)$ and $\|A y\|>2 b$, then $\alpha(A y)>b)$. Thus, we choose $y \in \mathfrak{P}(\alpha, b, c)$ such that $\|A y\|>2 b$. Then, from (3.7),

$$
\begin{aligned}
\alpha(A y) & =A y\left(\frac{1}{4}\right) \\
& =\int_{0}^{1} G\left(\frac{1}{4}, s\right) f(y(s)) d s \\
& =\int_{0}^{1} \frac{G\left(\frac{1}{4}, s\right)}{G\left(\frac{1}{2}, s\right)} G\left(\frac{1}{2}, s\right) f(y(s)) d s \\
& \geq \min _{0 \leq r \leq 1} \frac{G\left(\frac{1}{4}, r\right)}{G\left(\frac{1}{2}, r\right)} \int_{0}^{1} G\left(\frac{1}{2}, s\right) f(y(s)) d s \\
& =\frac{1}{2} A y\left(\frac{1}{2}\right) \\
& =\frac{1}{2}\|A y\| \\
& >b
\end{aligned}
$$


and (C3) of Theorem 2.1 is satisfied. Hence, an application of Theorem 2.1 completes the proof.

\section{EXTENSIONS TO HIGHER EVEN ORDER PROBLEMS}

We conclude this work by extending the results of Section 3 in obtaining three symmetric positive solutions for the boundary value problem,

$$
\begin{aligned}
& (-1)^{m} y^{(2 m)}-f(y)=0, \quad 0 \leq t \leq 1, \\
& y^{(2 i)}(0)=0=y^{(2 i)}(1), \quad 0 \leq i \leq m-1,
\end{aligned}
$$

where $f: \mathbb{R} \rightarrow[0, \infty)$ is continuous. Again, we will apply Theorem 2.1 We remark that, if $y \in C^{(2 m)}[0,1]$ is a solution of (4.1), (4.2), then $y$ is both nonnegative and concave on $[0,1]$.

In this context, Theorem 2.1 will be applied in conjunction with a completely continuous operator whose whose kernel is the Green's function for

$$
(-1)^{m} y^{(2 m)}=0
$$

satisfying (4.2). With $G(t, s)$ defined by (3.2), define

$$
G_{1}(t, s)=G(t, s),
$$

and for $2 \leq j \leq m$, iteratively define

$$
G_{j}(t, s)=\int_{0}^{1} G(t, r) G_{j-1}(r, s) d r .
$$

Then, $G_{m}(t, s)$ is the Green's function for (4.3), (4.2).

Let

$$
\begin{aligned}
& 0<e<\frac{1}{2}, \quad k_{m}=\left(\max _{0 \leq t \leq 1} \int_{0}^{t} G_{m}(t, s) d s\right)^{-1} \\
& =\left(\int_{0}^{1} G_{m}\left(\frac{1}{2}, s\right) d s\right)^{-1}, \quad l_{m e}=\left(\min _{e \leq t \leq 1-e} \int_{e}^{1-e} G_{m}(t, s) d s\right)^{-1} \\
& =\left(\int_{e}^{1-e} G_{m}(e, s) d s\right)^{-1} \quad \text { and } \\
& C_{m e}=\max _{0 \leq t \leq 1} \frac{G_{m}\left(\frac{1}{2}, t\right)}{G_{m}(e, t)} .
\end{aligned}
$$

Let the Banach space $\mathfrak{B}$, cone $\mathfrak{P} \subset \mathfrak{B}$ and concave functional $\alpha: \mathfrak{P} \rightarrow[0, \infty)$ be as in Section 3 , Let $A: \mathfrak{B} \rightarrow \mathfrak{B}$ be defined by

$$
A y(t)=\int_{0}^{1} G_{m}(t, s) f(y(s)) d s .
$$

Then an application of Theorem 2.1 yields the following result

Theorem 4.1. Let $0<a<b<K^{-1} c$ and suppose $f$ satisfies

(i) $0 \leq f(w)<k_{m} a$ for $0 \leq w \leq a$,

(ii) $f(w) \geq l_{m e} b$ for $b \leq w \leq K b$,

(iii) $0 \leq f(w) \leq k_{m} C$ for $0 \leq w \leq c$.

If $K \geq C_{m e}$, then the boundary value problem (4.1), (4.2) has three symmetric positive solutions $y_{1}, y_{2}$ and $y_{3}$ satisfying $\left\|y_{1}\right\|<a, b<\alpha\left(y_{2}\right)$, and $\left\|y_{3}\right\|>a$ with $\alpha\left(y_{3}\right)<b$. 
Proof. The argument is similar to that in the proof of Theorem 3.1 except we apply Theorem 2.1] with $d=K b$.

Remarks. 1. Theorem 3.1 corresponds to the special case $m=1, e=\frac{1}{4}$ of Theorem 4.1.

2. Theorem 3.1 improves on Theorem 7 of Avery [2] by requiring $f(w) \geq 16 b$ holds on $[b, 2 b]$ rather than on the longer interval $[b, 4 b]$.

3. Similarly, Theorem 3.1 improves on Theorem 3 of Henderson and Thompson [5] by requiring $f(w) \geq 16 b$ holds on $[b, 2 b]$ rather than on the longer interval $[b, 4 b]$.

4. Theorem 3.1 requires $c>2 b$ which is an improvement over Theorem 3 of [5] which requires $c>4 b$.

5. Set $l_{m}=\min _{0<e<\frac{1}{2}} l_{m e}=l_{m e *}$. This is the smallest lower bound $f(w)$ must satisfy in order to guarantee solutions exist using our Theorem 4.1 That the minimum is obtained can be seen as follows:

(i) $l_{m e}=\left(\min _{e \leq t \leq 1-e} \int_{e}^{1-e} G_{m}(t, s) d s\right)^{-1}$ is a continuous function of $e$,

(ii) $G_{m}(t, s)$ is a increasing function of $t$ on $[0,1 / 2]$ by 4.5 since $G(t, s)$ is a increasing function of $t$ on $[0,1 / 2]$ and $G_{m-1}(t, s)$ is nonnegative,

(iii) $l_{m e} \rightarrow \infty$ as $e \rightarrow 0^{+}$, since $G(0, s)=0$ so that $G_{m}(0, s)=0$, and hence $\int_{0}^{1} G_{m}(0, s) d s=0$, and

(iv) $l_{m e} \rightarrow \infty$ as $e \rightarrow(1 / 2)^{-}$, since $\int_{0}^{0} G_{m}(t, s) d s=0$.

6. Since $C_{m e}=\max _{0 \leq t \leq 1} \frac{G_{m}\left(\frac{1}{2}, t\right)}{G_{m}(e, t)}$ and $G_{m}(l, t)$ is an increasing function of $l$ on $[0,1 / 2]$ for each $t$ in $[0,1]$, it follows that $C_{m e *}=\max _{e * \leq e \leq 1 / 2} C_{m e}$. Thus, if we set $e=e *$ so that $l_{m}=l_{m e *}$ is the smallest lower bound $f(w)$ must satisfy, then the trade off is that the smallest constant $K$ we require in our Theorem 4.1. $C_{m e *}$, is maximised; if we choose $e \in(0, e *)$, then there is $\bar{e} \in(e *, 1 / 2)$ such that $l_{m \bar{e}}=l_{m e}$ and we can use $K=C_{m \bar{e}} \leq C_{m e *}$ in Theorem 4.1. In particular, if we choose $e=e *$, then the smallest interval on which the lower bound $l_{m}$ is required on $f(w),\left[b, C_{m e *} b\right]$, is maximised and the smallest lower bound we require on $c, C_{m e *} b$, is maximised.

The constants $k_{m}, l_{m e}$ and $C_{m e}$ are not easy to compute explicitly. We can estimate these using the following estimates:

$$
\begin{array}{ll}
0<G_{m}(t, s) \leq \frac{1}{6^{m-1}} s(1-s), & 0<t, s<1 \\
G_{m}(t, s) \geq \frac{11^{m-1}}{2^{7 m-5} 3^{m-1}} s(1-s), & \frac{1}{4} \leq t \leq \frac{3}{4}, 0 \leq s \leq 1 .
\end{array}
$$

From these estimates it follows that $k_{m} \geq 6^{m}, l_{m \frac{1}{4}} \leq \frac{2^{7 m} 3^{m}}{11^{m}}$ and $C_{m \frac{1}{4}} \leq \frac{2^{6 m-4}}{11^{m-1}}$. These estimates are good for $m=1$ but are not very good for $m>1$. It follows that in Theorem 4.1 we may replace (i), (ii) and (iii) by

(i) $)^{\prime} 0 \leq f(w)<6^{m} a$ for $0 \leq w \leq a$,

(ii) $)^{\prime} f(w) \geq \frac{2^{7 m} 3^{m}}{11^{m}} b$ for $b \leq w \leq \frac{2^{6 m-4}}{11^{m-1}} b$,

(iii) $)^{\prime} 0 \leq f(w) \leq 6^{m} c$ for $0 \leq w \leq c$ where $0<a<b<\frac{11^{m-1}}{2^{6 m-4}} c$. 


\section{REFERENCES}

[1] D. Anderson, Multiple positive solutions for a three-point boundary value problem, Mathematical and Computer Modelling 27 (1998), 49-57. MR 99b:34040

[2] R. Avery, Existence of multiple positive solutions to a conjugate boundary value problem, MSR Hot-Line, 2, No.1 (1998), 1-6. MR 98i:34034

[3] R. Avery and A. Peterson, Multiple positive solutions of a discrete second order conjugate problem, Pan American Mathematical Journal, 8 (1998), 1-12. MR 99i:39001

[4] D. Guo and V. Lakshmikantham, Nonlinear Problems in Abstract Cones, Academic Press, Boston, 1988. MR 89k:47084

[5] J. Henderson and H.B. Thompson, Existence of multiple solutions for some $n$-th order boundary value problems, preprint.

[6] R. Leggett and L. Williams, Multiple positive fixed points of nonlinear operators on ordered Banach spaces, Indiana University Mathematics Journal, 28 (1979), 673-688. MR 80i:47073

[7] Y. Sun and J.-X. Sun, Multiple positive fixed points of weakly inward mappings, Journal of Mathematical Analysis and Applications, 148 (1990), 431-439. MR 91f:47084

Department of Mathematics, Auburn University, Auburn, Alabama 36849-5310

E-mail address: hendej2@mail.auburn.edu

Centre for Applied Dynamical Systems, Mathematical Analysis and Probability, Department of Mathematics, The University of Queensland, Brisbane, Queensland 4072 Australia

E-mail address: hbt@maths.uq.edu.au 